\title{
BIBECHANA
}

ISSN 2091-0762 (Print), 2382-5340 (Online)

Journal homepage: http://nepjol.info/index.php/BIBECHANA

Publisher: Department of Physics, Mahendra Morang A.M. Campus, TU, Biratnagar, Nepal

\section{Effect of electron temperature in a magnetized plasma sheath using kinetic trajectory simulation}

\author{
R. Chalise ${ }^{1, *}$, S. K. Pandit ${ }^{1}$, G. Thakur ${ }^{1,2}$, R. Khanal ${ }^{2}$ \\ ${ }^{1}$ Department of Physics, Amrit Campus, Lainchaur, Tribhuvan University, Kathmandu, Nepal \\ ${ }^{2}$ Central Departments of Physics, Tribhuvan University, Kirtipur, Nepal \\ *Email: plasma.roshan@gmail.com
}

\section{Article Information:}

Received: May 30, 2020

Accepted: June 21, 2020

Keywords:

Plasma-wall transition

Sheath

Presheath

Bohm criterion

Kinetic simulation

\begin{abstract}
The understanding of the properties of magnetized plasma sheath has been various beneficial applications in surface treatment, electron emission gun, ion implantation, and nuclear fusion, etc. The effect of electron temperature on the magnetized plasma sheath has been studied for a fixed magnetic field and ion temperature. It has been observed that various plasma sheath parameters can be prominently altered by the varying temperature of the electron. The density of ion is influenced more by the change in electron temperature rather than the electron density. The temperature of the electron has a great effect at the wall, when electron temperature increases, the ion and electron densities at the wall decreases. This shows the potential at the wall also decreases follows the Poisson's equation. Similarly, the electric field also decreases but total charge density increases when the electron temperature is increased.
\end{abstract}

DOI: https://doi.org/10.3126/bibechana.v18i1.29204

This work is licensed under the Creative Commons CC BY-NC License. https://creativecommons.org/licenses/by-nc/4.0/

\section{Introduction}

The study of the interaction between plasma and the solid absorbing wall is almost very old as plasma science itself [1-3].The topic of magnetized plasma-wall Transition (PWT) has been extensively studied using different models in recent years because of its importance to magnetic fusion and other applications [4-8]. As the plasma comes into contact with the wall, it is bombarded by the fastmoving electrons and attains a negative potential w.r.t. the plasma. The negative potential then attracts positive ions towards the wall and repels the electrons. In the steady-state, a positive space charge region forms near the wall to balance the flow of the ions and electrons into the wall. The plasma boundary layer is thus divided into two separate regions: a neutral presheath adjacent to the plasma and a positively charged sheath adjacent to the wall. The sheath width is usually a few Debye lengths and its task is to shield the plasma from the wall, while the presheath width is determined by the size of the plasma container or by the ion mean 
free path [2]. R. Chodura studied the effect of a magnetic field on the transition layer between a plasma and an absorbing wall by developed a numerical model, which is used to simulate the motion of plasma particles in presence of electric and magnetic fields for a prescribed particle influx at the plasma boundary [1]. The transition layer proves to have a double structure comprising a quasineutral magnetic presheath preceding the electrostatic Debye sheath. The magnetic presheath scales with the ion gyroradius at the sound speed and with the angle of the magnetic field. The total electric potential drop between plasma and wall proves to be fairly insensitive to the magnitude and angle of the magnetic field.

B. Singha et al. carried out an experimental observation to see the grid biasing voltage in controlling the thickness in a system [3]. The experiment is carried out in a stainless steel chamber which is divided into two regions by a mesh grid. It has been observed that at both conditions of increasing and grid biasing voltage, the width expands in the region, whereas in the diffused region, no such noticeable variation has been found. B. Pandey et al. investigated the structure of the plasma sheath in the presence of an oblique magnetic field [9]. It is shown that the width of the plasma sheath is dependent not only on the collision frequencies and the plasma magnetization but also on the angle of magnetic field orientation. The size of the sheath layer decreases with the increase in the angle between the magnetic field and the wall. A. K. Shaw et al. studied the properties of a magnetized multicomponent plasma sheath with a finite positive ion temperature by using three fluid hydrodynamic models [10]. With the increase of positive ion temperature, the lighter positive ion density peaks increase only at the sheath edge and shift towards the sheath edge for both in the absence and presence of the magnetic field. For heavier positive ions, in the absence of a magnetic field, the density peaks increase at the sheath edge. But in the presence of a magnetic field, the density fluctuations increase at the sheath edge. For both cases, the density peaks shift towards the sheath edge. R. Chalise and R. Khanal extended the kinetic trajectory simulation (KTS) model to $1 \mathrm{~d} 3 \mathrm{v}$ cases and used to study the magnetized plasma-wall transition [11-12]. It was observed that the magnetized plasma sheath is characterized by three distinct regions: presheath, magnetic presheath, and electrostatic Debye sheath. R. Chaulagain et al. studied the effect of ion temperature in a magnetized plasma sheath, which consists of two species of positive ions using kinetic theory [4]. The physical parameters change slowly near the sheath entrance but exhibit a steep gradient near the wall. The effect of the applied magnetic field is more in ions whereas the electrons are almost nonresponsive and they are not influenced directly. T. Gyergyek and J. Kovačič used two-fluid models for the analysis of ion temperature effects and showed that the physically acceptable monotonic solutions are possible only when the ion velocity, electron velocity, and electric field at the sheath edge are beyond certain minimum values [5]. S. Adhikari et al. simulated bounded plasma with a spatially generated source in the presence of an oblique magnetic field [6]. The kinetic particle-in-cell technique has been used to track particles fully kinetically and the ions are observed to follow interesting dynamical behavior near the collector sheath. The low energetic ions reflect back to the ion source region at certain angles of inclination. The reflection seems to be prominent at a low angle of inclination. G. Sharma et al.numerically investigated the sheath structure and ion flux to the wall in a collisional magnetized plasma consisting of two temperature electrons [7]. It has been observed that sheath width and ion flux to the wall are not monotonous functions of collision frequency as well as hot electron concentration. The sheath edge velocity is found to decrease with the increase of hot electron concentration and hot electron temperature. In this work, the basic plasma parameters (ion density, electron density, electric potential, electric field, 
and total charge density) are studied in a magnetized plasma sheath for different electron temperatures, using the KTS model [11]. The model is recently modified to study the effect of presheath electron temperature on magnetized plasma-wall transition and wall sputtering for the case of plasma having two species of positive ions [8]. This work is of significance in all practical applications of magnetized plasma, and in particular in modeling of divertor in magnetic fusion devices, where the plasma comes into contact with a material wall. In tokamaks, a particle running away from the core plasma follows a particular magnetic field line to reach the divertor wall. Thus, we are interested to study the effect of electron temperature in the particle guided by a constant magnetic field and at a fixed obliqueness with the wall.

\section{Basic principle of KTS}

KTS is an iterative method for numerically calculating self-consistent, time-independent kinetic plasma states in some given bounded spatial region [11]. In order to obtain the distribution function at any point $(\vec{x}, \vec{v})$ in the phase-space, the related trajectories of phase-space are followed up to the point where the distribution function is known. The electron and ion velocity distribution functions at the sheath edge are assumed to be cut-off Maxwellian in such a way that the most important requirement of the presheath-sheath transition are satisfied, i.e. quasineutrality, the sheath-edge singularity condition, continuity of the first three moments of each species, and the kinetic Bohm criterion.

In the general case of time-dependent, collisional kinetic theory, the species-s velocity distribution function describes the Boltzmann equation:

$\frac{\mathrm{df}^{\mathrm{s}}}{\mathrm{dt}}=\left[\frac{\partial}{\partial \mathrm{t}}+\overrightarrow{\mathrm{v}} \cdot \nabla+\overrightarrow{\mathrm{a}}^{\mathrm{s}} \cdot \frac{\partial}{\partial \overrightarrow{\mathrm{v}}}\right] \mathrm{f}^{\mathrm{s}}=\mathrm{C}^{\mathrm{s}}(1)$

The kinetic Boltzmann equation (1) for collisionless cases takes the well-known from of Vlasov equation:

$$
\left[\frac{\partial}{\partial \mathrm{t}}+\overrightarrow{\mathrm{v}} \cdot \nabla+\overrightarrow{\mathrm{a}}^{\mathrm{s}} \cdot \frac{\partial}{\partial \overrightarrow{\mathrm{v}}}\right] \mathrm{f}^{\mathrm{s}}=0
$$

i.e. $\mathrm{f}^{\mathrm{s}}=$ constant. This means that the velocity distribution function is constant for an observer moving along a collisionless trajectory. Then, the electron and ion densities are given by

$$
n^{s}(\vec{r})=\int_{-\infty}^{+\infty} d^{3} v f^{s}(\vec{r}, \vec{v})
$$

The space charge density is defined as

$$
\rho(\vec{r})=\sum_{s} q^{s} n^{s}(\vec{r})
$$

The electrostatic potential $\varphi(\vec{x})$ is to be found from Poisson's equation

$$
\frac{\mathrm{d}^{2} \varphi(\overrightarrow{\mathrm{r}})}{\mathrm{dx}^{2}}=\frac{-\rho(\overrightarrow{\mathrm{r}})}{\varepsilon_{0}}
$$

and the electric field is given by

$$
E(\vec{r})=-\nabla \varphi(\vec{r})
$$

In KTS simulation, the equations (2 - 6) are solved along the collisionless trajectories for given initial conditions.

\section{Magnetized Plasma Sheath Model}

The $1 d 3 v$ model of magnetized plasma sheath considered for the present work adapted from the KTS method is shown schematically in Fig. 1.

The right-hand boundary $(x=L)$ is the "sheath entrance", which separates the non-neutral, collisionless sheath region $(x<L)$ from the quasineutral collisional presheath region $(x>L)$, and the left-hand boundary $x=0$ is an absorbing wall. An oblique magnetic field acts in the $x-y$ plane making angle ' $\theta$ ' with the $x$-axis, which is also the direction of the electric field:

$$
\mathrm{B}=\mathrm{B}_{0}[\cos \theta \hat{\mathrm{x}}+\sin \theta \hat{\mathrm{y}}]
$$

The plasma particles enter the sheath region, a region of interest, from the right-hand boundary with cut-off Maxwellian velocity distribution, and both the boundaries are perfectly absorbing. As the core plasma is quasineutral the particle distribution is Maxwellian and as the ions are accelerated towards the wall they become shifted Maxwellian with cut-off velocity so that the Bohm criterion is satisfied. 


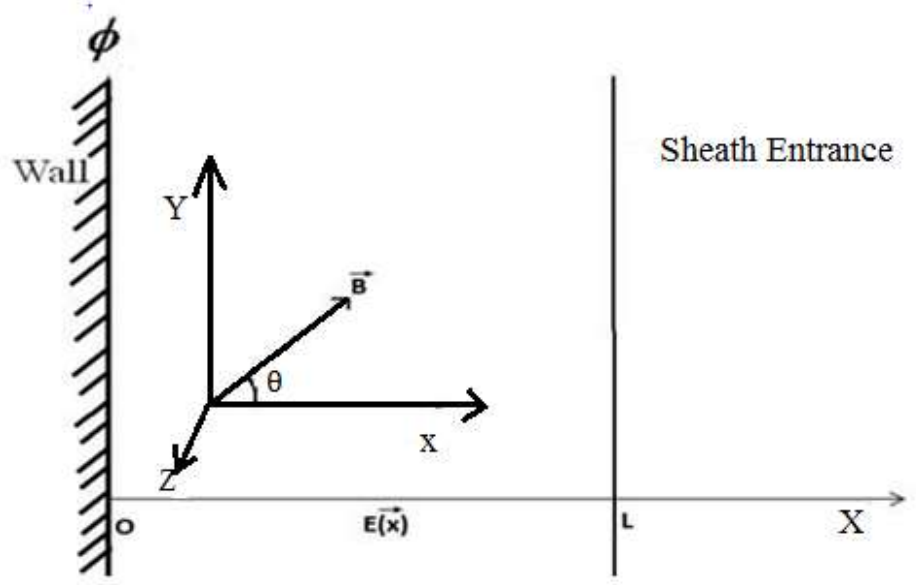

Fig. 1: Schematic diagram of the magnetized plasma sheath model [11].

On the other hand, electrons are reflected by the negative wall and hence their distribution is also cut-off. Accordingly, the electron velocity distribution function at the entrance is given by

$$
\mathrm{f}^{\mathrm{e}}(\mathrm{L}, \mathrm{v})=\mathrm{A}^{\mathrm{e}} \exp \left[-\left(\frac{\left(\mathrm{v}_{\mathrm{X}}^{2}+\mathrm{v}_{\mathrm{Y}}^{2}+\mathrm{v}_{\mathrm{Z}}^{2}\right)}{\left(\mathrm{v}_{\mathrm{tf}}^{\mathrm{e}}\right)^{2}}\right)+\frac{\mathrm{e} \varphi(\mathrm{x})}{\mathrm{k}_{\mathrm{B}} \mathrm{T}_{\mathrm{tf}}^{\mathrm{e}}}\right] \Theta\left(\mathrm{v}_{\mathrm{cL}}^{\mathrm{e}}(\mathrm{x})-\mathrm{v}_{\mathrm{x}}\right)
$$

where is $v_{c}^{e}(x)=\sqrt{\frac{2 e\left[\phi(x)-\phi_{0}\right]}{m^{e}}}$ the electron

cut-off velocity at $x, k_{B}$ is the Boltzmann constant, $\varphi(\mathrm{x}=0)=\varphi_{0}$ is potential at the wall and $\Theta(\mathrm{x})$ is the Heaviside function, i.e.

$$
\Theta(x)=\begin{aligned}
& 1 \text { if } x \geq 0 \\
& 0 \quad \text { elsewhere }
\end{aligned}
$$

The ion velocity distribution function at $\mathrm{x}=\mathrm{L}$ is given by

$\mathrm{f}^{\mathrm{i}}(\mathrm{x}=\mathrm{L}, \mathrm{v}<0)=\mathrm{A}^{\mathrm{i}} \exp \left[-\left(\frac{\left.\left(\mathrm{v}_{\mathrm{x}}-\mathrm{v}_{\mathrm{ml}}^{\mathrm{i}}\right)^{2}+\mathrm{v}_{\mathrm{y}}^{2}+\mathrm{v}_{\mathrm{z}}^{2}\right\}}{\left(\mathrm{v}_{\mathrm{tf}}^{\mathrm{i}}\right)^{2}}\right)\right] \Theta\left(\mathrm{v}_{\mathrm{cl}}^{\mathrm{i}}-\mathrm{v}_{\mathrm{x}}\right)$

where $\quad v_{\mathrm{tf}}^{S}=\sqrt{\frac{2 k_{B} T^{s}}{m^{s}}}$ is the species- $s$ thermal velocity, $v_{\mathrm{mL}}^{i}$ is the ion "Maxwellian-maximum" velocity at $\mathrm{x}=\mathrm{L}$ and $v_{\mathrm{cL}}^{i}\left(v_{\mathrm{cL}}^{i}<0\right)$ is the ion cut off velocity at $x=L$.
For the velocity distribution, given by equation (8) the electron density at $\mathrm{x}=\mathrm{L}$ can be evaluated using equation (3):

$$
\mathrm{n}^{\mathrm{e}}=\frac{\mathrm{A}^{\mathrm{e}} \pi^{3 / 2} \mathrm{v}_{\mathrm{tf}}^{\mathrm{e}^{3}}}{2} \mathrm{C}^{\mathrm{e}}
$$

where

$$
\mathrm{C}^{\mathrm{e}}\left(\mathrm{T}_{\mathrm{f}}^{\mathrm{e}}, \varphi_{0}\right)=1+\operatorname{erf}\left(\mathrm{v}_{\mathrm{cL}}^{\mathrm{e}} v_{\mathrm{tf}}^{\mathrm{e}}\right)=1+\operatorname{erf} \sqrt{\frac{-2 \mathrm{e} \varphi_{0}}{\mathrm{kT}^{\mathrm{e}}}}
$$

"erf" represents the "error function";

$$
\operatorname{erf}(\mathrm{x})=\frac{2}{\sqrt{\pi}} \int \mathrm{d} \xi \operatorname{erf}\left(-\xi^{2}\right)
$$

Now from the velocity distribution function (10) and (3), the ion density is

$$
\mathrm{n}_{\mathrm{L}}^{\mathrm{i}}=\frac{\mathrm{A}^{\mathrm{i}} \pi^{3 / 2} v_{\mathrm{tf}}^{\mathrm{i}^{3}}}{2} \mathrm{C}^{\mathrm{i}}
$$

where

$$
\mathrm{C}^{\mathrm{i}}\left(\mathrm{T}_{\mathrm{f}}^{\mathrm{i}}, \varphi_{0}\right)=1+\operatorname{erf}\left(\tau_{\mathrm{cL}}^{\mathrm{i}}\right)
$$

and

$$
\tau_{\mathrm{cL}}^{\mathrm{i}}=\left(\frac{\mathrm{v}_{\mathrm{cL}}^{\mathrm{i}}-\mathrm{v}_{\mathrm{mL}}^{\mathrm{i}}}{\mathrm{v}_{\mathrm{tf}}^{\mathrm{i}}}\right) .
$$

\section{Results and Discussion}

The ion density versus distance from the wall in the sheath region for a magnetic field of $300 \mathrm{mT}$ at $\theta=45^{0}$ for different electron temperatures (between $9.5 \mathrm{eV}$ to $13.5 \mathrm{eV}$ ) at the sheath entrance is shown in Fig. 2. In this, and all similar plots, the distance is normalized w.r.t. the electron Debye length at the 
sheath entrance. From the plot, it is observed that the ion density decreases slowly initially and close to the wall it decreased rapidly with a sharp gradient close to the wall due to the Debye shielding property of the plasma. The ion density at the sheath region decreases as the electron temperature is increased. The increase in electron temperature causes the thermal velocity of ions to increase, and hence, to maintain the particle flux conservation, the ion density decreases.

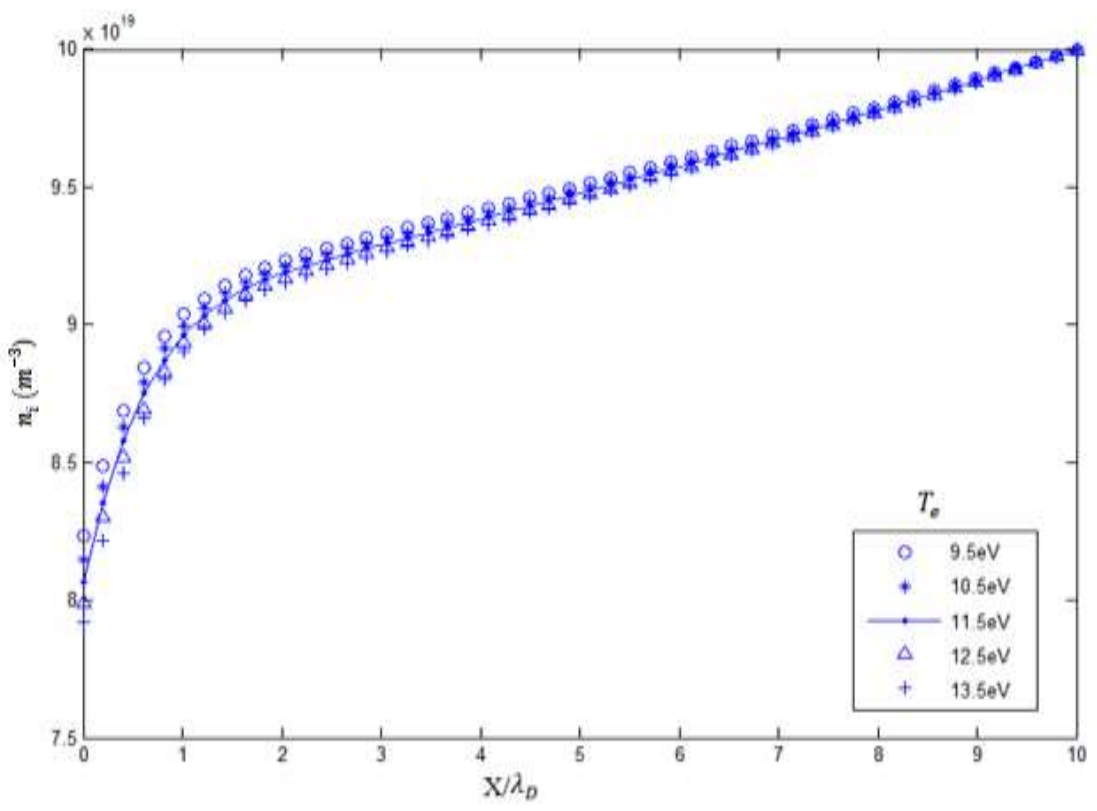

Fig. 2: Ion density profile for different electron temperatures.

Fig. 3 shows the ion density reaching the wall versus electron temperatures of the plasma for both unmagnetized $(\mathrm{B}=0)$ and magnetized $(\mathrm{B}=300$ $\mathrm{mT}$ ) cases. In absence of the magnetic field, the ions move straight away from the entrance guided by the electric field that increases as they move towards the wall. In the presence of the magnetic field, they start gyrating and their motion takes a longer time to move away from the entrance, where the electric field has a nominal effect compared to the magnetic field. This argument is in agreement with earlier reported works based on fluid approach $[1,2]$. This will result in a decrease of ion densities at the wall as shown by red line in Fig. 3. It is observed that the ion density decreases systematically with increasing the value of electron temperatures. The ion density, whose value at the entrance is $10^{20} \mathrm{~m}^{-3}$, decreases to $8.2 \times 10^{19} \mathrm{~m}^{-3}$ and $7.9 \times 10^{19} \mathrm{~m}^{-3}$ at the wall when the electron temperatures at the entrance are $9.5 \mathrm{eV}$ and $13.5 \mathrm{eV}$ respectively.

The electron density profile versus normalized distance from the wall for a magnetic field of 300 $\mathrm{mT}$ at $\theta=45^{0}$ for different electrons temperatures is shown in Fig. 4. It has been observed that the electron density decreases slowly, initially and the decrement is rapid close to the wall, where a sharp gradient is formed. As the temperature is increased the thermal velocity of electrons also increases, accordingly to maintain the flux conservation, electron density must decrease.

To compare the particle densities and also to visualize the breakdown of quasineutrality in the sheath region both the ion and electron density profiles are plotted in Fig. 5, for a magnetic field of $300 \mathrm{mT}$ at $\theta=45^{\circ}$ and the electron temperature at the sheath entrance $T_{\mathrm{ps}}^{e}=9.5 \mathrm{eV}$. As already 
discussed, both the densities decrease towards the wall from the sheath entrance, the electron density decreasing much faster than that of ions. While at the sheath entrance the two densities are equal, the ion density exceeds the electron density by almost

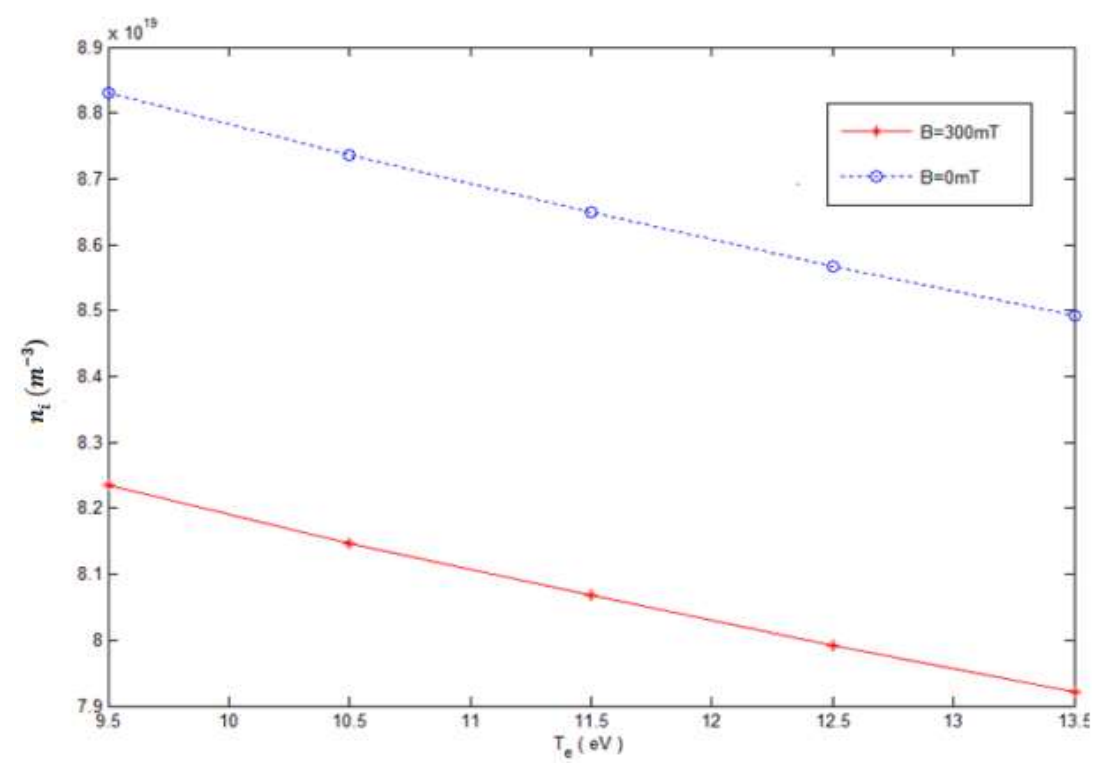

Fig. 3: Ion density at the wall versus electron temperature for unmagnetized and magnetized cases.

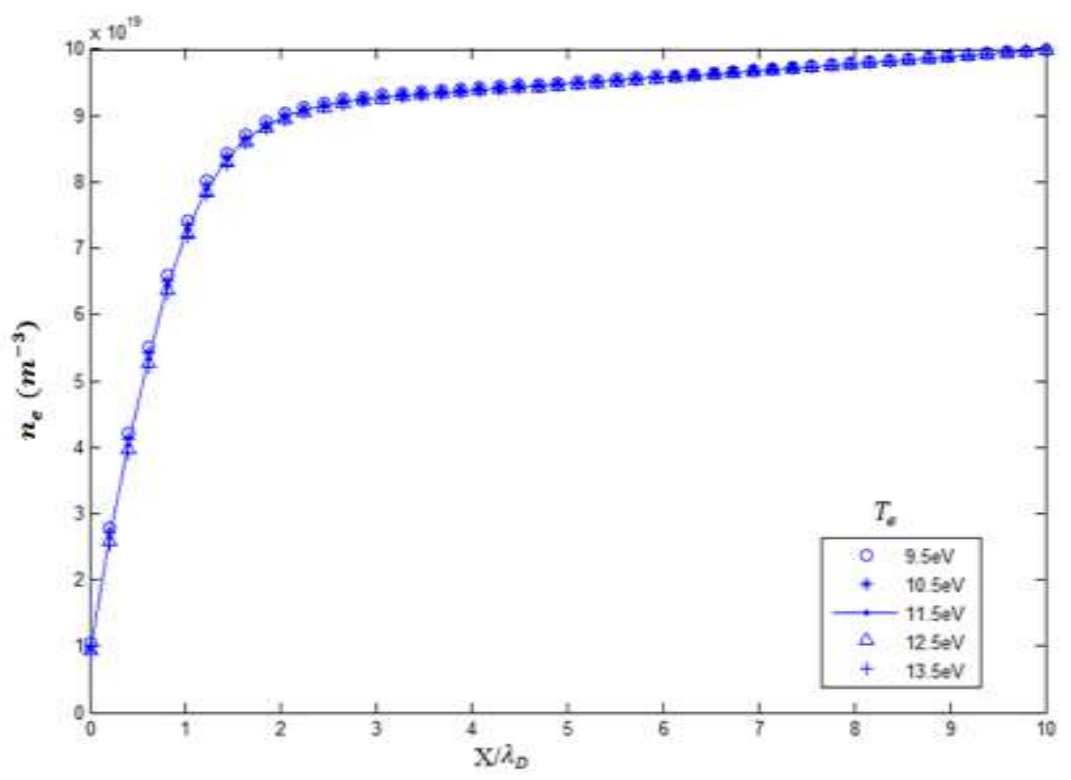

Fig. 4: Electron density profile for different electron temperatures. 


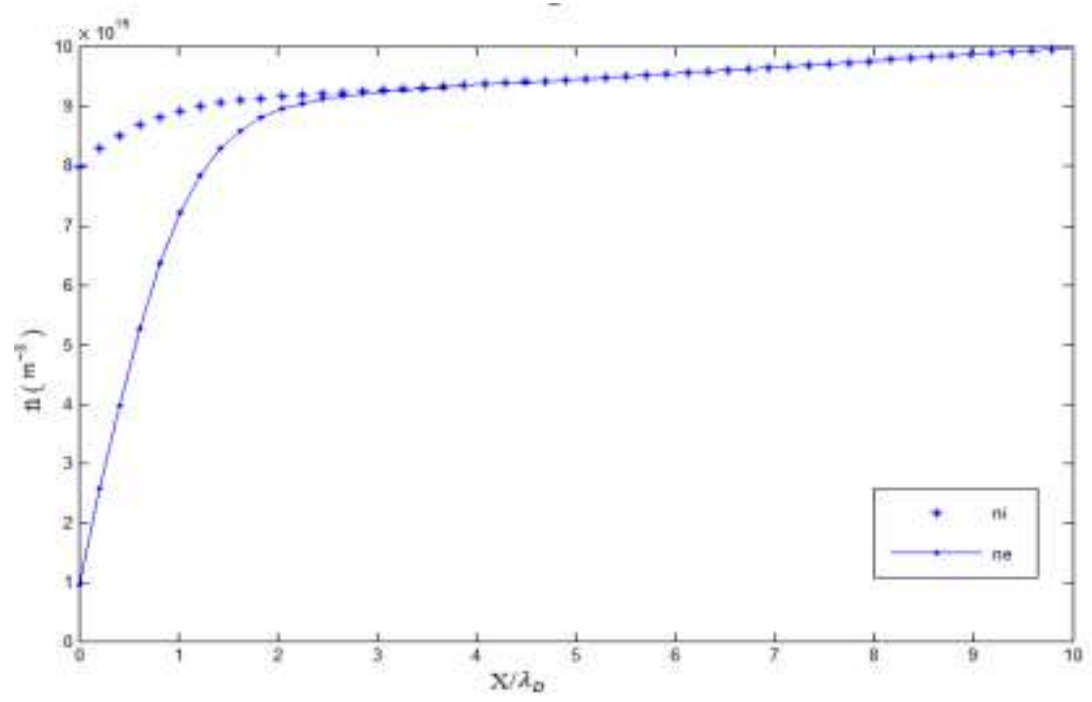

Fig. 5: Ion and electron density profiles for $9.5 \mathrm{eV}$ electron temperature.

Fig. 6 shows the potential versus normalized reach the wall much faster than at lower distance from the wall for a magnetic field of 300 temperatures.

$\mathrm{mT}$ at $\theta=45^{0}$ for different electron temperatures. It Fig. 7 shows the electric field profile versus is observed that the electric potential decreases normalized distance from the wall at a magnetic slowly at first and much faster close to the wall, field of $300 \mathrm{mT}$ at $\theta=45^{\circ}$ for different electrons where it shows a sharp gradient. The decrement of electric potential is consistent with the variation of ion and electron densities (Fig. 5). It is also observed that on increasing the electron temperature the wall will be more negative since more electrons temperatures. From the plot, it is observed that the electric field decreases monotonically in the sheath region and is almost constant except close to the wall, where it decreases rapidly as expected from earlier plots.

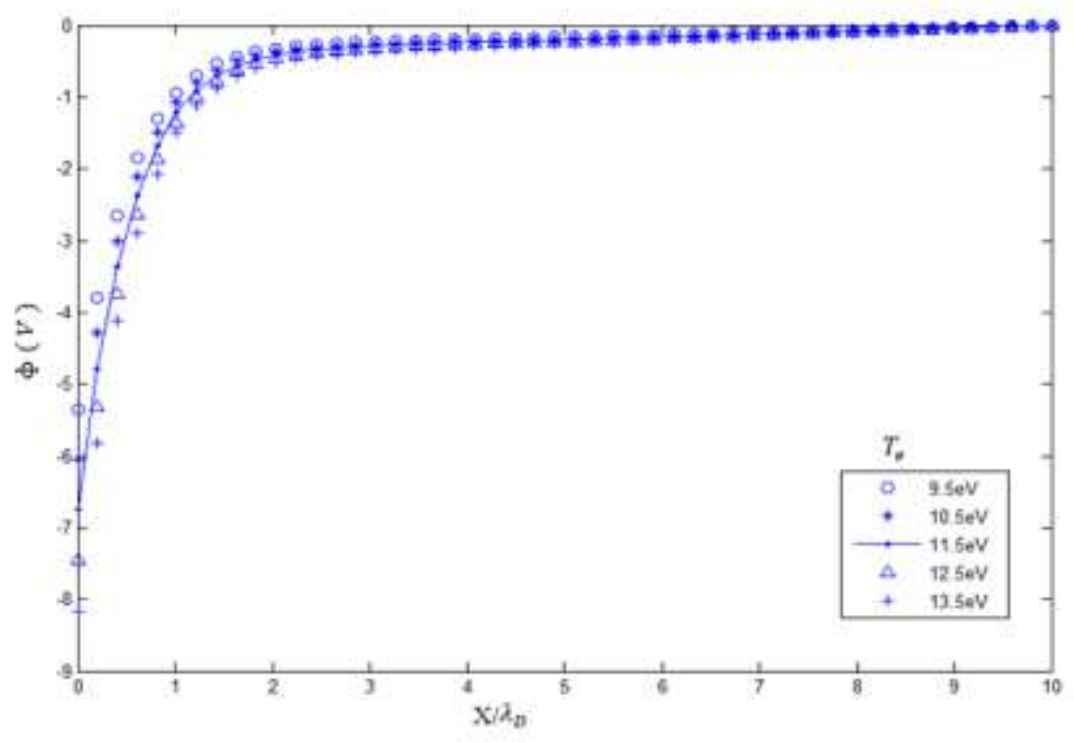

Fig. 6: Potential profile for different electron temperatures. 


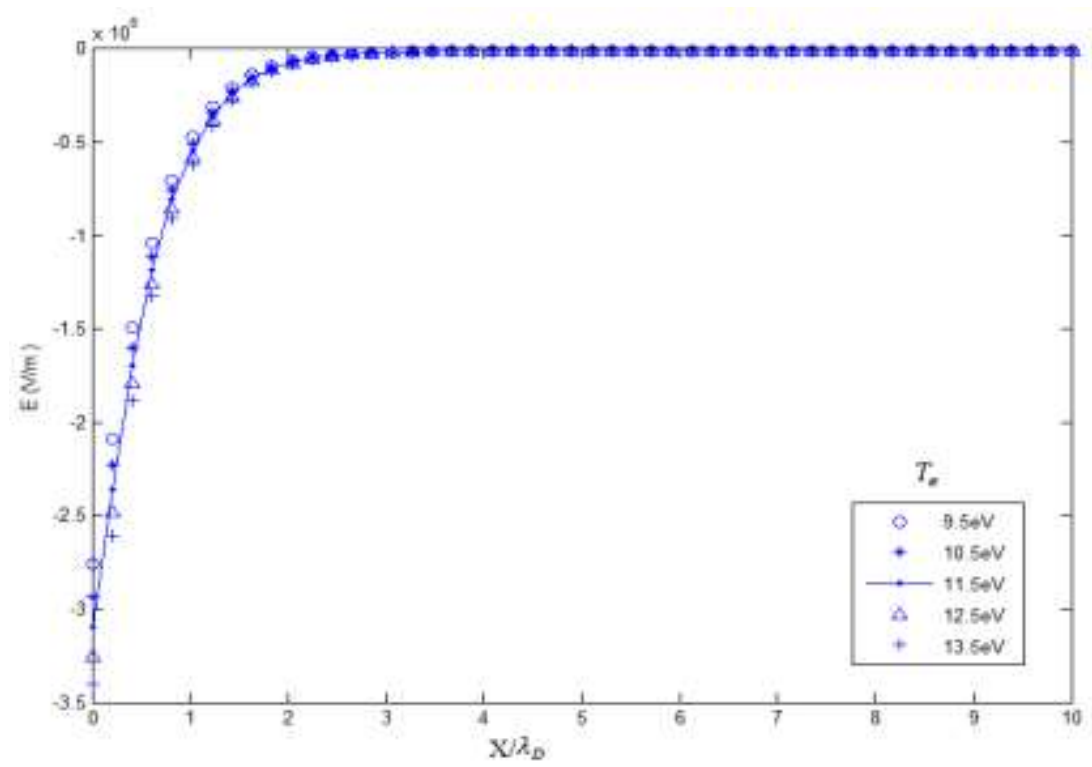

Fig. 7: Electric field profile for different electron temperatures.

\section{Conclusion}

The magnetized plasma sheath for different electron temperatures at the sheath entrance for a uniform external magnetic field was studied using the Kinetic Trajectory Simulation (KTS) method. It has been observed that various plasma sheath parameters (ion density, electron density, potential, electric field, and total charge density) are significantly affected by varying temperature of the electrons. The ion density is highly influenced by the change in electron temperature rather than the electron density. When electron temperature increases the ion and electron densities at the wall decreases, and this results in a decrease in the values of the potential and electric field as well. The obtained results agree well with earlier reported works on unmagnetized and magnetized cases [4, 11, 12]. These results suggest that the electron temperature can be a useful parameter that can be altered to control the particle and fluxes reaching the wall. On the other hand, it is possible to calculate what value of electron temperature at sheath entrance is required to have desired values of particle densities, potential, electric field, and total charge density at the wall. This provides a basis of controlling particle and energy fluxes in both ways; to control their flow (e.g., in applications requiring plasma confinement away from the wall), or to allow the particles to reach the wall (in plasma processing of materials).

\section{References}

[1] R. Chodura, Plasma-wall transition in an oblique magnetic field, Phys. Fluids 25 (1982) 1628-1633. http://dx.doi.org/10.1063/1.863955

[2] K. U. Riemann, The Bohm criterion and sheath formation, J. Phys. D: Appl. Phys. 24 (1991) 493518. http://dx.doi.org/10.1088/0022-3727/24/4/001

[3] B. Singha, A. Sarma and J. Chutia, Influence of magnetic field on plasma sheath and electron temperature, Review of Scientific Instruments 72 (2001) 2282-2287. http://dx.doi.org/10.1063/1.1362437

[4] R. Chaulagain, R. Chalise and R. Khanal, Effect of ion temperature variation in two-ion species magnetized plasma sheath, J. Materials Science and Engineering A 7 (2017) 320-326. 
http://dx.doi.org/10.17265/2161-6213/2017.11$\underline{12.007}$

[5] T. Gyergyek and J. Kovačič, Numerical analysis of ion temperature effects to the plasma-wall transition using a one-dimensional two-fluid model. II. Asymptotic two-scale limit, Phys. Plasmas 24 (2017) 063506 (18pp).

http://dx.doi.org/10.1063/1.4984787

[6] S. Adhikari, R. Moulick and K. S. Goswami, Ion dynamics in a magnetized source-collector sheath, Phys. Plasmas 25 (2018) 094504 (8pp).

http://dx.doi.org/10.1063/1.5045186

[7] G. Sharma, S. Adhikari, R. Moulick, S. S. Kausik and B. K. Saikia, Effect of two temperature electrons in a collisional magnetized plasma sheath, Physica Scripta 95 (2020) 035605 (9pp). http://dx.doi.org/10.1088/1402-4896/ab5548

[8] S. Basnet, A. Sarma and R. Khanal, Effect of presheath electron temperature on magnetized plasma-wall transition and wall sputtering by plasma having two species of positive ions, Physica Scripta 95 (2020) 065601 (11pp). http://dx.doi.org/10.1088/1402-4896/ab7b89
[9] B. P. Pandey, A. Samarian and S. V. Vladimirov, Plasma sheath in the presence of an oblique magnetic field, Plasma Phys. Controlled Fusion 50 (2008) 055003 (10pp).

http://dx.doi.org/10.1088/0741-3335/50/5/055003

[10] A. K. Shaw, S. Kar and K. S. Goswami, The positive ion temperature effect in magnetized electronegative plasma sheath with two species of positive ions, Phys. of Plasma 19 (2012) 102108 (8pp). http://dx.doi.org/10.1063/1.4759460

[11] R. Chalise and R. Khanal, A kinetic trajectory simulation model for magnetized plasma sheath. Plasma Phys. Control. Fusion 54 (2012) 095006 (5pp). http://dx.doi.org/10.1088/0741-3335/54/9/095006

[12] R. Chalise and R. Khanal, Self consistent one dimension in space and three dimension in velocity kinetic trajectory simulation model of magnetized plasma-wall transition, Phys. Plasmas 22 (2015) 1135005 (5pp). http://dx.doi.org/10.1063/1.4934601 